\title{
The Simulation and Analysis of Quantum Illumination Radar Based on Labview
}

\author{
Huifang $\mathrm{Li}^{1, \text { a }}$, Minghui Zhang ${ }^{1, \mathrm{~b}}$, Kaibin Wang ${ }^{2, \mathrm{c}}$, \\ ${ }^{1}$ School of Electronics and Information, Northwestern Polytechnical University, Xi'an, China \\ ${ }^{2}$ Xi'an Research Institute of China Coal Technology \& Engineering Group Corp \\ aLihuifang@nwpu.edu.cn, ${ }^{b}$ nikkoch@163.com, ${ }^{\mathrm{C}}$ wangkaibin@cctegxian.com
}

Keywords: quantum illumination; quantum radar; quantum entanglement, Labview .

\begin{abstract}
In this paper, the concept and principle of quantum radar are introduced in detail. First The Quantum radar mode using entangled photons is discussed, and the simulation scheme of quantum illumination is designed. Then, the principle of each module is analyzed, and simulation programs are finished with Labview. Finally, it is demonstrated by experiments that the simulation platform can is useful to the analysis of quantum radar effectively.
\end{abstract}

\section{Introduction}

Quantum radar [1] is a new concept of radar based on photon quantum properties to solve existing technology bottleneck of classic radar in the aspect of measurement, detection and imaging. Since the Defense Advanced Research Projects Agency (DARPA) made the Quantum Sensor Program [2] in 2007 to research a new generation radar using quantum technology, quantum radar has become an attractive research area because of its superior performance. In 2008, Allen gave a design schematic of radar systems and the methods using entangled quantum particles [3]. At present there are three kinds operating principle of quantum radar is presented. Among them, Quantum illumination is the most promising technology [4]. Due to the physical implementation of quantum illumination radar is difficulty and high cost, based on physical experiments structure of Italian scientists ED Lopaeva prototype, we established quantum detection simulation system in this paper with Labview, then we give a description and discussion about each module of the system in detail.

\section{The Principle of Quantum Radar}

Entangled photons quantum radar works as shown in Fig.1 [5], an entangled pair is produced in a quantum radar, one of the photons is sent towards the target by transmitter while the other is kept in the system. The targets reflect the photon which is subsequently detected by the receiver. The information of targets will be acquired through the correlation between the photons reflected back and the photons kept in the system. The correlation can be exploited to increase the performance of the device. One of the advantages of quantum radar is the fact that some targets appear to look bigger when looked with low photon number pulses.

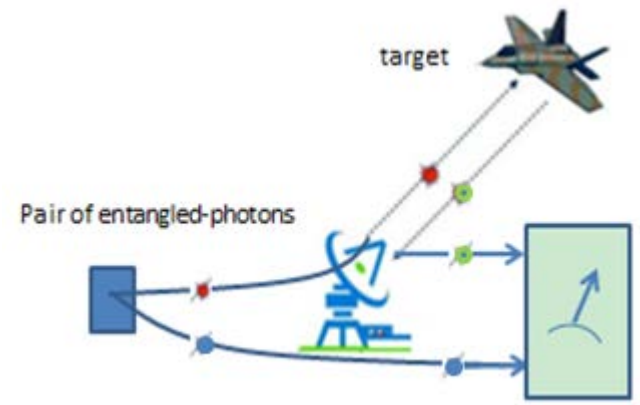

Fig.1 The radar system of entangled photons quantum 
If a target is illuminated by a quantum illumination device, the goal is to detect the presence of the target even in a noisy and lossy environment. The interaction between the signal photons and the target can be modeled as a beam splitter with small reflectivity $\eta$.Noise is injected into the system with average photon number $b$ into each optical mode. We can assume that $b<<1$ to simplify the mathematical formalism. This case corresponds to the scenario where the thermal radiation is substantially below the signal photon energies.

If the photo detector is characterized by a bandwidth $\mathrm{W}$ and a temporal detection window $\mathrm{T}$, then, in principle, the device can distinguish between d different radiation modes [1]:

$\mathrm{d} \approx \mathrm{W} \times \mathrm{T}$

Furthermore, we assume that $\mathrm{T}$ is small enough so that at most one noise photon is observed per detection event, that is:

$\mathrm{d} \times \mathrm{b}<<1$

Quantum illumination radar using entangled photons will be simulated and analyzed in following.

\section{Simulation Scheme}

In 2013, Italy scientist E.D.Lopaeva firstly achieved the experiment of quantum illumination [6]. The setup for entangled photons, designed by E.D.Lopaeva, is shown in fig.2.

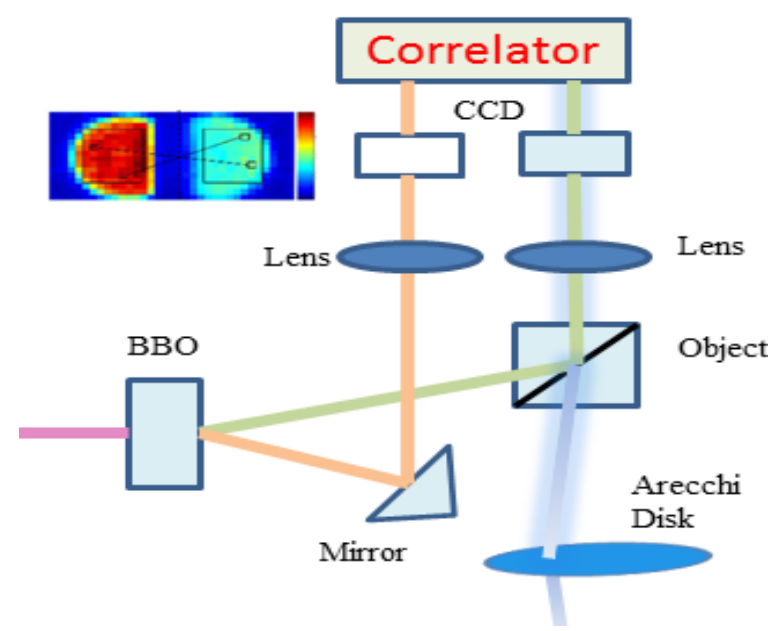

Fig.2 the simulation scheme of quantum illumination

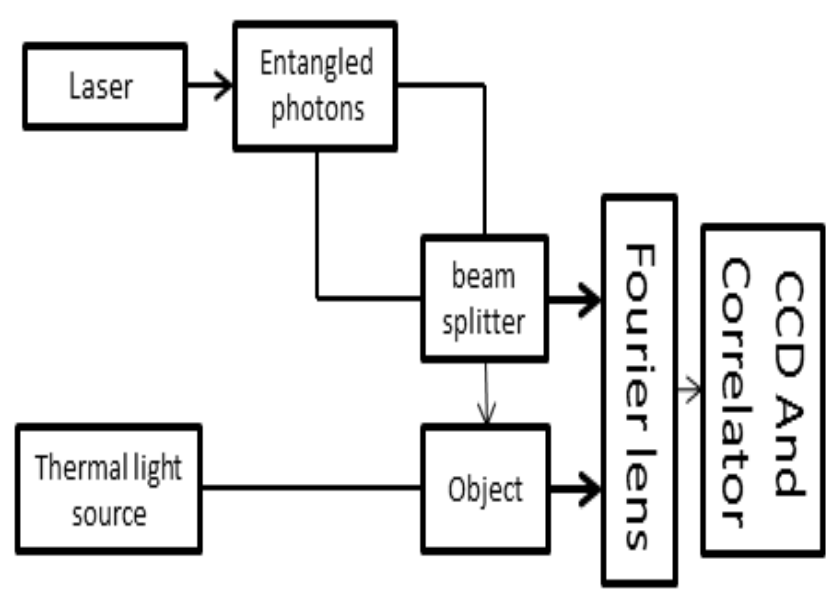

Fig.3 The experimental scheme of quantum illumination by E.D.Lopaeva

In the Fig. 2, the power of the pump is adjusted to obtain the same energy of the after the BBO crystal. Parametric Down Conversion (PDC) is exploited to generate two correlated light emissions. One beam is directly reflected towards the detection system, while a target object (a 50:50 beam splitter) is posed on the path of the other one, where it is superimposed with a thermal background produced by scattering a laser beam on an Arecchi's rotating ground glass. The lens, placed at the focal length from the crystal and the CCD camera, realizes the Fourier transform of the field at the output face of the crystal. The CCD cameras detect photons. When the object is removed, only the background reaches the detector. When the object (actually a beam splitter) is present, the correlated beam is partially detected, together with the thermal field from the Arecchi's disk, otherwise it is lost.

Based on above experimental principle, our aim is to lead the quantum illumination idea to an experimental demonstration in a simulated condition with Labview. Fig.3 show the simulation scheme of quantum illumination. Here, an unknown background, and a reception strategy based on photon-counting detection and second-order correlation measurements are considered in our simulation. On the one hand our approach maintains most of the appealing features of the original idea, like a huge quantum enhancement and a robustness against noise. On the other hand, our study also provides a significant example of ancilla-assisted quantum protocol. 


\section{The Model and Simulation of Each Module}

The laser source. The description of the spatial structure of the different beam modes has been object of theoretical and experimental analysis since the invention of the laser, All higher-order beams, such as Hermite-Gaussian, Laguerre-Gaussian and Bessel beams, can elegantly be derived by acting with differential operators on the plane wave representation of the fundamental Gaussian mode. Laguerre-Gaussian beam can transfer orbital momentum to a trapped particle, inducing it to rotate around the optical axis. For this reason, Laguerre-Gaussian optical beam can be easily generated laser beam entangled on orbital angular momentum with SPDC. Laguerre Gaussian laser beam modes electric fields, which are defined by a cylindrical coordinate system, are expressed as

$$
E(r, \phi, z)=\frac{A_{p l} \omega_{0}}{\omega(z)}\left(\sqrt{2} \frac{r}{\omega(z)}\right)^{l} L_{p}^{l}\left(2 \frac{r^{2}}{\omega(z)^{2}}\right) \exp \left(-\frac{r^{2}}{\omega(z)^{2}}\right) \cdot \exp \left\{-i\left[k\left(z+\frac{r^{2}}{2 R}\right)-(2 p+l+1) \tan \frac{z}{z_{0}}\right]\right\}\left\{\begin{array}{l}
\cos (l \phi) \\
\sin (l \phi)
\end{array}\right.
$$

Where $\omega=\omega_{0} \sqrt{1+\left(z / z_{R}\right)^{2}}$ is the spot radius in transmission distance for $\mathrm{z}$.

$Z_{0}=\pi \omega_{0}^{2} / \lambda$ is Rayleigh distance.

$\lambda$ is wavelength of light.

$k=2 \pi / \lambda$ denotes wavenumber.

From the Eq.3, we can complete VI program of the two beam as shown in figure 4 . The intensity is denoted by real part, the phase is denoted by imaginary part.

The production of the entangled photons. Spontaneous parametric down-conversion (also known as SPDC) is one of an important process in quantum optics which is used to product a source of entangled photon pairs. In the process of producing entangled photon pairs, on the basis of the law of conservation of energy and law of conservation of momentum, a nonlinear crystal is used to split photon beams into pairs of photons that have combined energies and momenta equal to the energy and momentum of the original photon and crystal lattice. Splited photon beams are phase-matched in the frequency domain, and have correlated polarizations.

In the process of the photon pairs generated in spontaneous down-conversion are entangled in orbital angular momentum (OAM). The corresponding mode functions are naturally expressed in terms of eigenstates of the paraxial OAM operator. Thus, one can decompose the quantum state $|\psi\rangle$ in the base of the eigenstates of the OAM operator, as

$$
|\psi\rangle=\sum_{l_{1} p_{1}} \sum_{l_{2} p_{2}} C_{l_{1}, p_{2}}^{l_{2}, p_{1}}\left|l_{1}, p_{1}\right\rangle\left|l_{2}, p_{2}\right\rangle
$$

Where $\left|l_{1}, p_{1}\right\rangle$ correspond to the signal mode, $\left|l_{2}, p_{2}\right\rangle$ correspond to the idler mode, and the amplitude $C_{I_{2}, p_{2}}^{l_{1}, p_{1}} C_{1_{2}, p_{2}}^{l_{1}, p_{1}}$ is written as

$$
C_{l_{2}, p_{2}}^{l_{1}, p_{1}}=\int d q_{s} d q_{i} \phi\left(q_{s}, q_{i}\right)\left[L G_{p_{1}}^{l_{1}}\left(q_{s}\right)^{*} L G_{p_{2}}^{l_{2}}\left(q_{s}\right)^{*}\right]
$$

Based on Eq.5, the probability distribution can be realized with VI as a Program module.

Finally, the experimental scheme of quantum illumination including detector, as outlined in the scheme of Fig. 1, is simulated with the VI module. As shown Fig.4. In the simulation the detected object is a 50:50 beam splitter (BS) embedded in a thermal modes. Charge-coupled device (CCD) are placed in each axis of the signal beam and reference beam behind lens.

Fig.6 represents the measurement scheme of the signal to noise ratio (SNR). It is based on following formula:

$$
S N R=\frac{\left|\overline{\operatorname{Cov}_{1,2+}-\operatorname{Cov}_{1,2-}}\right|}{\sqrt{\overline{\delta^{2}\left(\operatorname{Cov}_{1,2+}\right)}+\overline{\delta^{2}\left(\operatorname{Cov}_{1,2-}\right)}}}
$$




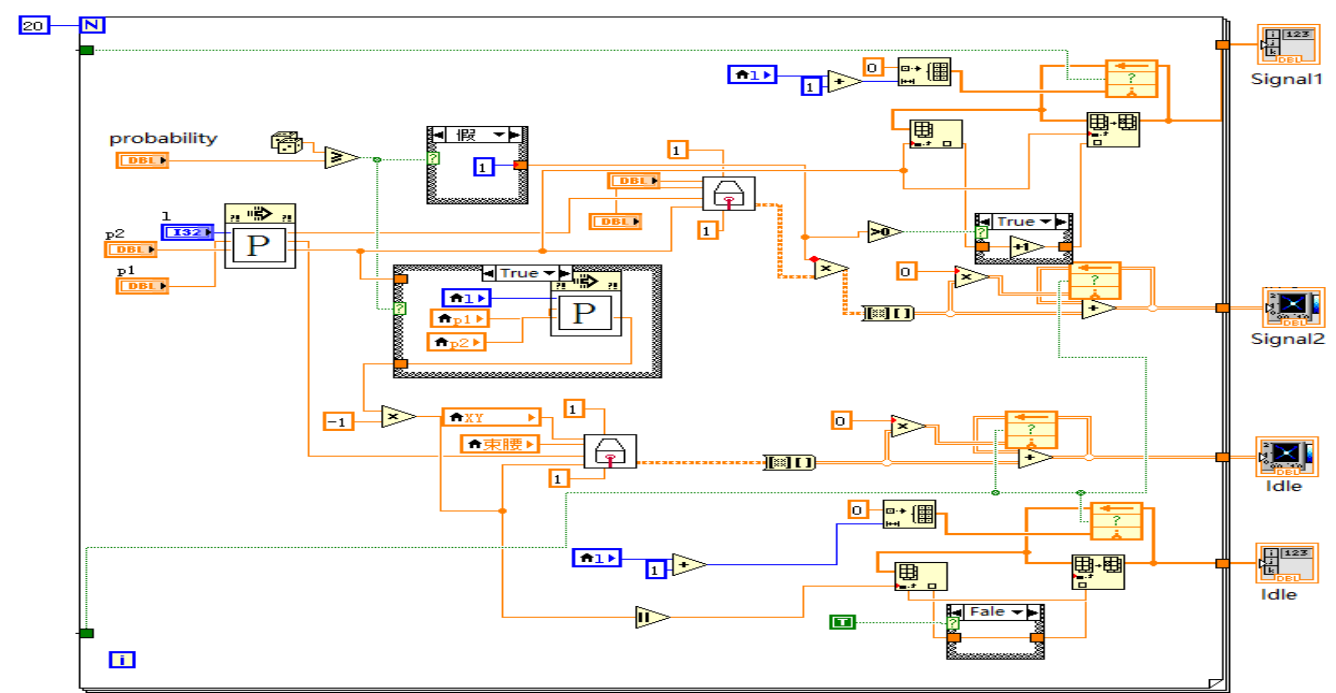

Fig.4 The simulation experiment of quantum illumination including detector Where $\operatorname{Cov}_{1,2+}$ is matrix of covariance that the target exist, and $\operatorname{Cov}_{1,2-}$ is one that there is no the target.
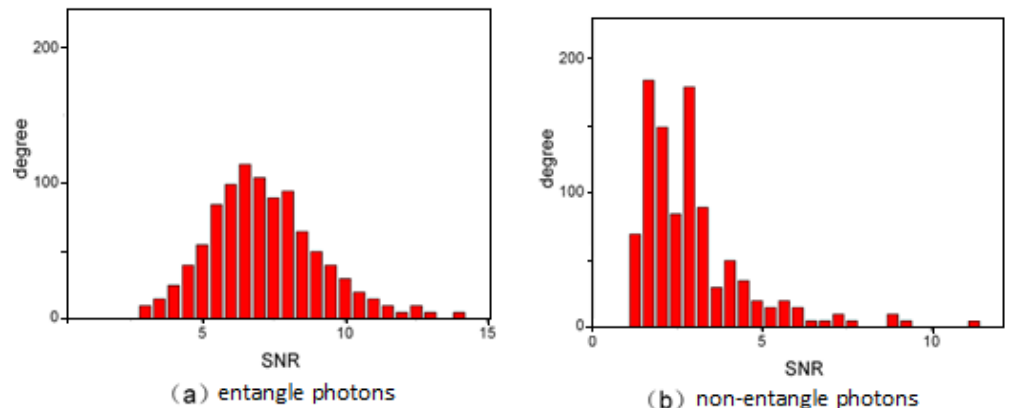

Fig.5 SNR comparison

Fig.5 displays SNR comparisons between entangle photons and non-entangle photons. The parameter values used in simulation are the number of background noise $=256$, the Standard deviation $=8$, the number of photon $=256$, the times of repetition experiment $=1000$.

In fig.5 (a) (for entangle photons), mean value is 6.85, the Standard deviation is 2.47. In fig.5 (b) (for non-entangle photons), it is random distribution between 1 and 6 , without obvious distribution regularity.

\section{Conclusions}

In this paper, based on the principal of quantum illumination radar, The Mathematical Modeling of the laser source, entangled photons and the measurement of SNR and so on was established with LABVIEW. Then, the SNR comparisons between entangle photons and non-entangle photons is given. We made a detailed research on the performance of quantum illumination radar.

\section{References}

[1] MarcoLanzagorta: Quantum Radar (Morgan Claypool Publishers, USA, 2012).

[2] G. Burdge, G. Deibner, J. Shaprio et al.:Quantum Sensors Program,"DTIC Document 2009.

[3] E. H. Allen and M. Karageorgis, ed: Google Patents (2008)

[4] S.-H. Tan, B. I. Erkmen, V. Giovannetti, S, et al.: Physical review letters, Vol. 101(2008), p.253

[5] S. Lloyd: Science, Vol. 321 (2008), p.1463

[6] E. Lopaeva, I. R. Berchera, I. Degiovanni: Physical Review Letters, Vol. 110 (2013), p.153

[7] A. V. Kuznetsova: ICATT, 2013, Odessa, Ukraine, p.518. 